\title{
Technology of drinking water preparation using the reactor - clarifier
}

\author{
Yuriy Skolubovich ${ }^{1, *}$, Aleksander Skolubovich ${ }^{1},{\text { Evgeniy } \text { Voitov }^{1} \text { and Inna Makarikhina }}^{1}$ \\ ${ }^{1}$ Novosibirsk State University of Architecture and Civil Engineering (SIBSTRIN), Turgeneva str., \\ 159, 630008, Novosibirsk, Russia
}

\begin{abstract}
Siberian surface water and groundwater are characterized by low temperatures for a long year period. Many groundwater sources' organic composition are formed of soil and peat humus, marsh feeding of rivers, decomposition of plankton, higher water, and soil grass in reservoirs and lakes. Organic colloids in natural waters and in humic substances give the color of water. It's yellowish coloration of varying intensity. Thus, the Om River's water color in the city of Kuibyshev in the Novosibirsk Region is 500 degrees with feculence of less than $3 \mathrm{mg} / \mathrm{l}$. A number of underground water sources also have an increased content of organic contaminants caused by peat bogs at great depths and high water colority with low turbidity. For example, the water color is up to 1500 degrees in the Namtsy village of the SAHA-Yakutia Republic. In addition, underground water and, to a greater extent, surface water are often characterized by a high content of iron (up to $20 \mathrm{mg} / \mathrm{l}$ ), manganese (up to 4 $\mathrm{mg} / \mathrm{l}$ ), and other impurities of natural and anthropogenic origin. Iron and manganese are in natural waters in the form of mineral or organic complex compounds of humic or some fatty acids. In the second case, these waters are with increased oxidizability and rather aggressive nature. In particular, the iron content is $3 \mathrm{mg} / \mathrm{l}$, manganese is $1 \mathrm{mg} / \mathrm{l}$, the permanganate oxidizability is $50 \mathrm{mg} / \mathrm{l}$ in the water of the Om River.
\end{abstract}

\section{Introduction}

Traditional technologies for natural water preparation into drinking water are not designed to purify the original water with color over 120 degrees. [1]. The fact is the supersaturation degree of water by the coagulants hydrolysis products at their usual doses in the metastable zone is insufficient for the solid phase nuclei in the free volume of septic tanks at low temperatures. With the increase viscosity in water, kinetic mobility decrease in the process of colloidal particles hydrolysis and frequency of their collisions that cause agglomeration, coagulation purification of water proceeds unsatisfactorily.

With small water feculence, the process of spatial structure formation is also difficult in clarifiers with a suspended layer, due to the continuous movement of sediment's individual loose flakes in them.

\footnotetext{
* Corresponding author: michmacha@mail.ru
} 
In addition, humic substances sorbed on the surface of the coagulating hydroxide impart stability, preventing sorption during colored waters treating.

The use of contact clarifiers with a single-stage purification scheme or contact prefilters in front of the fast filters by a two-stage scheme for high-colored waters treatment in connection with high doses of coagulant is associated with their frequent flushing and large consumption of washing water, which reduces the useful productivity of water treatment plants.

The problem of low-temperature, low-turbidity, high-colored waters preliminary cleaning is partially solved by the use of clarifiers-recirculators, where a part of the formed sediment from the suspended layer is ejected into the initial water and their flakes serve as additional centers for condensation of the hydrolysis products. This allows increasing the productivity of clarifiers by $30-60 \%$ and to process water with color up to $300 \mathrm{deg}$. However, the rate of purified water flow does not exceed $0.8 \mathrm{~mm} / \mathrm{s}$ and the performance of the clarifier-recirculator remains low at low temperatures and turbidity of the initial water up to $20 \mathrm{mg} / 1$ [2].

In order to improve the coagulation processes in low-colored waters processing, the initial water is made turbidity with various materials, for example clay. The artificial particles introduction play the role of additional centers of condensation's hydrolysis products, promotes coagulation. The flocculating effect of polyelectrolytes also increases. The turbidity of the treated water leads to a weighting of coagulated suspension flocs, their hydraulic size and the effect of water purification increasing. However, the supply of material, that meets sanitary and technical requirements, preparation, dispensing of turbidity suspension, removal and disposal of phyto-slime that is formed, significantly increases the cost of the water treatment facilities operation.

\section{Materials and Methods}

New clarifier reactors were developed in NSUACE (Sibstrin) in order to improve the work efficiency and the facilities for the preliminary reagent cleaning of low-temperature, lowturbidity, high-color surface, as well as iron-manganese-containing groundwater for drinking and technical water supply, on the principle of contact coagulation, [3]. (RF patent No. 2307075)

The clarifying reactor (CR) for water purification works as follows (Fig. 1). Through the water supply line for clarification 3 , the initial water treated with reagents is supplied to an air separator 15 that prevents air bubbles from entering suspended contact mass layer and through the distribution standpipe 16 evenly distributed over the area of a device's clarification zone. Then, the clarified water passes from the bottom up through the contact mass layer 2 at a rate ensuring its minimal fluidization. At the same time, stagnant zones, which are present near the places of particles contact in the stationary state of the layer, are eliminated and the porosity of the charge is increased. Quartz sand or any other fine-grained material that meets the requirements for mechanical strength and chemical resistance is used as a contact mass. Catalytic acceleration of the of coagulant's hydrolysis process occurs, contact coagulation of the hydrolysis products, the adsorption of metal hydroxides by weighed contact mass grains, and the formation in the sediment layer, which itself is a sorbent for impurities of water by the volume concentration of the solid phase increasing in the suspended layer. Part of the sludge flakes is removed from the layer, retained by the thin-layer module 12, slides down the inclined walls of its rings and settles on the surface of the contact mass. As a result, a high water purification effect and a lower consumption of reagents are achieved. The device productivity is increased. The clarified water is collected by chute 4 and removed through the pipe 5 from the device. Contact mass is flushed after the protective action expiration of the weighed contact mass and the deterioration of 
clarified water quality in the following sequence. The gate valve on the tap of the washed water outlet 6 is opened, and on the discharge line of the clarified water 5 is closed. The valves are opened on the pipeline 17 connecting the ejector for pulp 8 to the lower part of the device body and to the supply pipeline 7 to the washing water ejector 8 . The clarified water is supplied through the pipeline 3 and the air separator 15 and the dipleg 16 to the contact mass 2. Washed water is supplied into the ejector 8. Contaminated contact weighed mass in the form of pulp through the pipeline 17 from the lower part of the device case is transported into the ejector pulp supply 8 to the upper part of the device body through pipe 9 and tangentially introduced into the semi-immersion cylinder 13 below through the air ejector 14 water level at an angle to its surface.

The intake of the contact mass in the ejector 8 is regulated by means of a valve installed on the pipeline 17 . The air introduced into the pulp with the aid of the ejector 14 is released into the water in the bubbles form and water-air rinsing of the contact mass occurs. It is enhanced by the separation of the sediment particles and contact-weight grains in the centrifugal force field in semi-submerged cylinder 13. Washed grains of the contact mass are deposited in a suspended layer, and sediment is discharged with the spent washed water. The contact mass removal in the collecting chute 4 is prevented by the thin-layer module. After the washing, the valves are closed on the pipelines 17, 7, 6. At the same time, the washing water supply to the ejector of the pulp 8 is stopped and the device continues to purify the water again. The contact weight to be weighed is used repeatedly during the cleaning process. The pulp is discharged from it via conduit 1 , if it is necessary to change the contact weight of the weighed mass or the emptying of the water purification device.

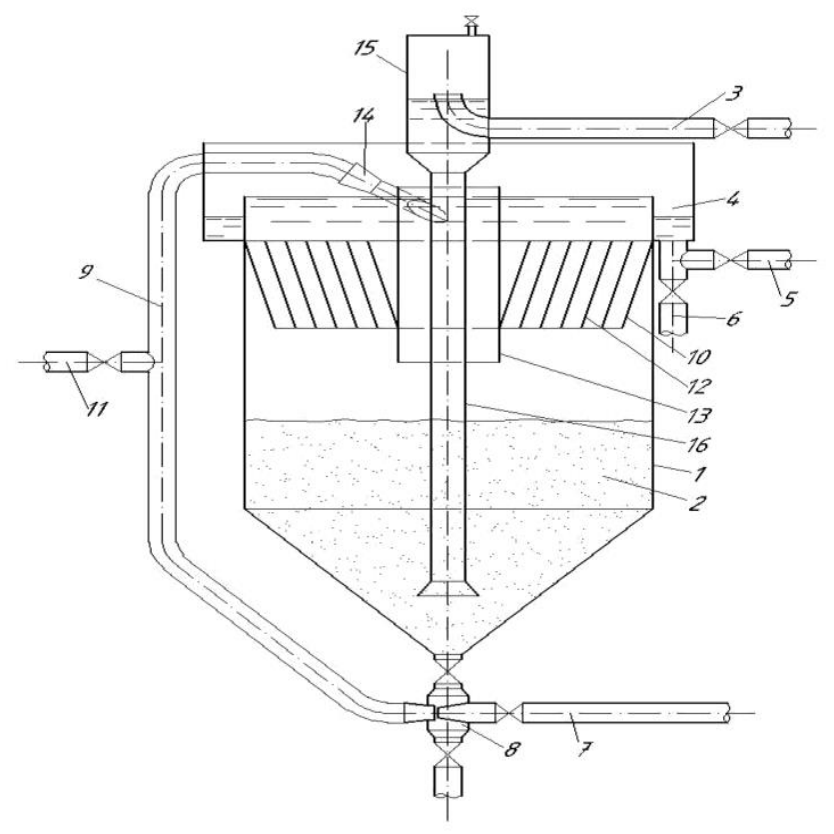

Fig. 1. Scheme of the clarifier reactor, where: 1 - clarifier reactor shell; 2 - contact weighed mass; 3 pipeline of source water; 4 - collecting channel of clarified and washing water; 5 - pipeline of clarified water; 6 - drainage line for washing water; 7 - pipeline for the supply of washing water to the hydroelevator; 8 - hydroelevator; 9 - pipeline for the transport of contact mass pulp; 10 - conical diaphragm; 11 - pipeline for the release of contact mass pulp; 12 - conical thin-layered module; 13 semisubmissive cylinder; 14 - air ejector of tangential input of pulp into a semi-submersible cylinder; 15 - air separator; 16 - lower distribution pipe; 17 - the pipeline for supplying the contact mass to the hydroelevator. 
Experimental studies of the reactor-clarifier were carried out at the water treatment plant of Kuibyshev NSR. High-quality low-water water of Om River was subjected to purification. The technological scheme structure of water purification consisted consistently of the following main structures: a clarifier reactor, an early granular filter, a sorption filter.

\section{Results}

An experimental model clarifier reactor intended for pre-clarification and discoloration of water had a diameter of $100 \mathrm{~mm}$ and a height of $3 \mathrm{~m}$. The reactor was structurally in accordance with the above dinitialing and loaded with a contact mass (CM) from homogeneous quartz sand with a particle size of 0.3-0.5 mm. The height of the dense loading layer was $1.1 \mathrm{~m}$. The model of the fast filter intended for further water purification consisted of three short pressure columns with a height of $0.6 \mathrm{~m}$ connected in series and loaded with crushed albitophyre with an average grain size of $2.5 ; 1.8$ and $1 \mathrm{~mm}$ in the first, second and third columns, respectively. Each column modeled a separate filter layer. The sorption filter model, where dissolved organic and chemical contaminations were extracted, and the final water purification took place, consisted of a single pressure head loaded with active carbon of grade AG-3, fractions 1-2.5 mm with a layer of $1 \mathrm{~m}$ height.

The observations results of one of the characteristic cycles of water clarification on a clarifying reactor, rapid grains and sorption filters are below.

The color of the river water was 130 degrees, turbidity $2 \mathrm{mg} / 1$, temperature $2^{\circ} \mathrm{C}$. The water was pretreated with solutions of coagulant of aluminum oxychloride with $20 \mathrm{mg} / 1$ dose, cationic flocculant Prastol 650 TR with a dose of $0.5 \mathrm{mg} / 1$ and introduced into the device through an air separator, dip tube.

With the upward movement of water, $25 \%$ weighing of the CM layer took place. The air separator prevented the air bubbles suctioning into the device and the weighted layer stability of the contact mass.

At the initial clarification moment, a circulation motion of $\mathrm{CM}$ grains was observed along the entire height of the layer from top to bottom. The formation of a main part of aluminum hydroxide precipitate took place from the water, sorption on humic particles sediment, accompanied by discoloration of water to the drinking norm (up to $20^{\circ} \mathrm{C}$ ) in the $\mathrm{CM}$ layer. A part of the sediment, which was not in the contact mass layer, in the form of large dense flakes, was excreted in the thin-layer module, drained down and settled on the surface of the contact mass layer. Clarified water, containing small flakes of sediment, came from the device for final cleaning on the fast filter model by a granular charge from crushed albitophyre.

As structured sediment of aluminum hydroxide is formed in a weighted contact mass, the adhesion of individual sediment structures and the fixation of $\mathrm{CM}$ grains among themselves, the weighed load layers gradually go upwards from bottom to top. The quality of the treated water did not change at the same time. After 6 hours of clarification, the load and all of its individual grains took a stably stationary position. The process of water clarification in a suspended but immobile layer of CM has become similar to the process of conventional filtration in the granular layer with the ascending movement of the water being purified. The velocity of the ascending stream on the clarifier reactor model was 1.8 $\mathrm{mm} / \mathrm{s}$. After 60 hours of clarifier reactor operation, the quality of the clarified water gradually began to deteriorate both in terms of the water color and its turbidity. At $5 \mathrm{mg} / 1$ of water turbidity, the clarification cycle was terminated due to the inadvisability of this quality water supply to the second stage of purification, the fast filter. During the 60-hour water clarification cycle on the reactor, subsequent water purification on the fast filter and sorption on the charcoal filter, based on the control analyzes results of the Kuibyshev 
Sanitary and Epidemiological Surveillance Laboratory, ensured the water quality corresponding to San. 2.1.4.1074-01.

At the end of each water treatment cycle, an ejection washing of the reactor-clarifier contact mass was carried out according to the above procedure. The specific consumption of the water ejector's working fluid was from 5 to $8 \mathrm{~m}_{3} / \mathrm{m}_{3}$ of sand. The accumulation of residual contaminants in the contact mass, controlled by the initial losses of pressure, was not observed during the semiannual period of the experimental studies, which indicates a high efficiency of washing the clarifier reactor.

The parameters of the clarifier reactor operation, obtained as a result of technological modeling, allowed calculating and optimizing its design and technological parameters.

The research results were used to design the production reactors-clarifiers in the development of the project for the reconstruction of the pump-filtering station in Kuibyshev.

\section{Conclusions}

1. The positive role of the granular medium lies in the fact that the flocculation processes take place in a closed pore space, where the collision probability of coagulated suspension particles significantly increases, and both stick to each other and to previously formed aggregates with clarification of low- turbid, colored waters, weakly mineralized waters with long periods of low temperatures. With the same quality of clarified water, the load on the clarification reactors can be increased by $2-3$ times in comparison with conventional clarifiers with a layer of suspended sediment. This allows them to be placed in tight spaces of reconstructed clarifiers without buildings expansion.

2. Grains of the contact mass are additional centers of flocculation and shift part of the coagulation process from the spatial structure formation that occurs in conventional clarifiers, to autocatalytic coagulation, sorption and adhesion. As a result, the strength and density of newly formed flocs and, correspondingly, their hydraulic size increase.

3. The clarification process in the contact mass layer is similar to the process of contact coagulation in a granular filter load. However, the grains of the weighed contact mass participate in the process of coagulation and sorption by the whole surface, in contrast to filters which grains have "dead" non-working zones at their contact points, they reduces the actual porosity of the filter load and the performance of the filters.

4. The use of clarification reactors for water pre-treatment makes it possible to reduce the use of reagents to $50 \%$.

5. The bulk of hydroxides flakes and impurities of water is retained in the clarifier reactor, which allows reducing the pollution load on the fast filters and doubling their productivity.

\section{References}

1. Russian Federation Standard SNiP 2.04.02-84

2. Recommendations on the application of water purification technology on clarifiers with a suspended layer of recirculated sludge (Households, Leningrad, 1985)

3. Yu.L. Skolubovich, Preparation of drinking water from underground sources (NGASU (Sibstrin), Novosibirsk, 2005) 